\title{
Composición y abundancia de comunidades microbianas asociadas al biofloc en un cultivo de tilapia
}

\author{
Microbiology community composition and abundance associated to biofloc in tilapia aquaculture
María del C. Monroy-Dosta ${ }^{1}$, Ramón De Lara-Andrade ${ }^{1}$, Jorge Castro-Mejía ${ }^{1}$, Germán Castro-Mejía ${ }^{1}$ y Mauricio G. Coelho-Emerenciano ${ }^{2}$

${ }^{1}$ Universidad Autónoma Metropolitana-Xochimilco, Departamento El Hombre y su Ambiente, Calzada del Hueso No. 1100, Col. Villa Quietud, 04960 México DF, México. monroydosta@hotmail.com

${ }^{2}$ Universidade do Estado de Santa Catarina, UDESC, Centro de Educação Superior da Região Sul, CERES, Rua Cel. Fernandes Martins No. 270, Progresso, CEP 88.790-000, Laguna, Santa Catarina, Brasil

\begin{abstract}
The goal of this study was to identify and estimate the abundance of microorganisms associated to biofloc culture system, developed from a 'macrocosm-microcosm' system type. In first section (cylinder 1000 L), 75 juveniles tilapias were inoculated with $5.0 \pm 0.95 \mathrm{~cm}$ mean length and $4.2 \pm 1.08 \mathrm{~g}$ mean weight were placed. Daily, the fishes were fed with commercial diet, maintaining a $\mathrm{C} / \mathrm{N}=15: 1$ ratio, carbon (molasses and rice dust) and nitrogen (from commercial feed) supply was controlled. This culture condition was maintained during 14 weeks. Several microorganisms associated to the flocs were identified using conventional microbiological tests. To estimate the abundance of organisms associated to flocs, a stereoscopic and optical microscopes were used. Both microscopes were connected to an interphase program for images counting (Image ProPlus v.7.0). The results shown changes in abundance of different microorganisms communities associated to flocs during 14 weeks of experiment. The main groups found in flocs were: bacteria, algae, ciliates, rotifers and nematodes. The results confirm that the bioflocs contributes significantly as in situ natural food source, because a great number of organisms may be associated to them including heterotrophic microbial communities like genus Sphingomonas, Pseudomonas, Bacillus, Nitrospira, Nitrobacter and yeast Rhodotorula sp. The literature show that these microorganisms favoring water quality and physiological good health at organisms in culture.
\end{abstract}

Key words: Bacteria, ciliates, flocs, nematodes, rotifers

Resumen.- El objetivo de este trabajo fue identificar y estimar la abundancia de microrganismos asociados a un sistema de cultivo biofloc desarrollado a partir de un sistema del tipo 'macrocosmo-microcosmo'. En la primera sección (cilindro de $1000 \mathrm{~L}$ ), se colocaron 75 tilapias con una longitud aproximada de 5,0 $\pm 0,95 \mathrm{~cm}$ y un peso promedio de 4,2 $\pm 1,08 \mathrm{~g}$. Los peces fueron alimentados diariamente con una dieta comercial, manteniendo una relación $\mathrm{C} / \mathrm{N}=15: 1$, mediante el aporte controlado de carbono (melaza y pulido de arroz) y nitrógeno (proveniente del alimento comercial). Esta condición fue mantenida durante 14 semanas. Se identificaron los diversos microrganismos asociados a los flóculos, utilizando pruebas microbiológicas convencionales. Se determinó la abundancia de los organismos asociados a los flóculos utilizando microscopio óptico y estereoscópico, ambos conectados con el programa de imágenes y de conteo (Image ProPlus v.7.0). Los resultados obtenidos indican cambios en la abundancia de las diversas comunidades de organismos asociados a los flóculos durante las 14 semanas del experimento. Los principales grupos encontrados fueron: bacterias, microalgas, ciliados, rotíferos y nematodos. Los resultados confirman que los flóculos contribuyen como fuente de alimento natural in situ, debido a que gran cantidad de organismos pueden estar asociados incluyendo comunidades microbianas heterótrofas de los géneros Sphingomonas, Pseudomonas, Bacillus, Nitrospira, Nitrobacter y la levadura Rhodotorula sp. La bibliografía menciona que estos microorganismos favorecen la calidad del agua y el bienestar fisiológico de los organismos en cultivo.

Palabras clave: Bacterias, ciliados, flóculos, nematodos, rotíferos 


\section{INTRODUCCIÓN}

En las últimas décadas dentro del sector acuícola, se han diseñado una serie de sistemas de producción para el cultivo de diversos organismos acuáticos, orientados a disminuir la utilización del agua y del espacio, aumentando considerablemente la densidad de cultivo (Timmons et al. 2002, Hargreaves 2006). Un ejemplo interesante de este tipo de sistemas, es el denominado biofloc, el cual consiste en el desarrollo de flóculos microbianos formados a partir de una alta relación carbono:nitrógeno en el agua, con poco o nulo recambio y alta oxigenación (Avnimelech 2012, Emerenciano et al. 2013), en los cuales se utilizan dietas con bajo contenido de proteína cruda (Azim \& Litle 2008) o fuentes de carbono externo tales como melaza (caña de azúcar) salvado de arroz, salvado de trigo, entre otros (Emerenciano et al. 2012), lo que permite el crecimiento de una comunidad microbiana, sobretodo de bacterias heterótrofas, que metabolizan los carbohidratos y toman nitrógeno inorgánico (principalmente $\mathrm{NH}_{4}$ ), reduciendo sus niveles y mejorando la calidad del agua (Crab et al. 2009).

Por otra parte, asociados a estos flóculos se han observado microalgas, zooplancton, coloides, polímeros orgánicos, cationes y células muertas que son consumidas por las especies cultivadas como fuente de proteína, por lo que los costos de alimentación se reducen en más del 25\% (Avnimelech 2006, De Schryver et al. 2008, Ekasari et al. 2010). En relación a técnicas para la caracterización de los microorganismos en sistemas Biofloc, Ray et al. (2010), señalan 3 métodos: microscopia, epiflorescencia y cromatografía de gases. La técnica más utilizada es la visual mediante microscopia, la cual permite determinar los principales grupos de microorganismos en los flóculos (Newall et al. 2006). La identificación de los componentes microbianos vinculados a los flóculos se reporta, sobre todo a nivel de grupo, sin llegar a género debido principalmente a la falta de precisión en la identificación. De acuerdo con Emerenciano et al. (2012) la calidad nutricional del biofloc puede variar substancialmente de 12 a 49 y 13 a $46 \%$ de proteína cruda y lípidos, respectivamente. La misma tendencia puede ocurrir con los niveles de PUFA y HUFA (Azim \& Litle 2008, Ekasari et al. 2010), estas variaciones pueden ser resultado de una diferente relación C:N, intensidades de luz, salinidad y sobretodo, de la conformación de la microbiota.

Por lo anterior, es necesario realizar estudios que además de conocer la composición microbiana, permitan identificar los diversos grupos a lo largo del cultivo para determinar la diversidad de alimento vivo disponible para los peces y camarones, por lo que el objetivo de esta investigación fue identificar la comunidad microbiana en un cultivo biofloc y los cambios que se presentan en su composición a lo largo de un ciclo de cultivo.

\section{Materiales y Métodos}

\section{DISEÑO EXPERIMENTAL}

Para llevar al cabo el experimento se diseñó un sistema del tipo 'macrocosmo-microcosmo' (Wasielesky et al. 2006, Emerenciano et al. 2011), que consistió de 2 secciones: la primera con un contenedor de fibra de vidrio circular de $1000 \mathrm{~L}$ de capacidad (macrocosmo), con un difusor de aire en el centro para garantizar el movimiento continuo y resuspensión de partículas; y la segunda sección formada por 5 tinas circulares de 250 L cada una (microcosmos) (Fig. 1), ambas unidades conectadas con un sistema de recirculación por medio de 2 bombas eléctricas de $1.0 \mathrm{HP}$.

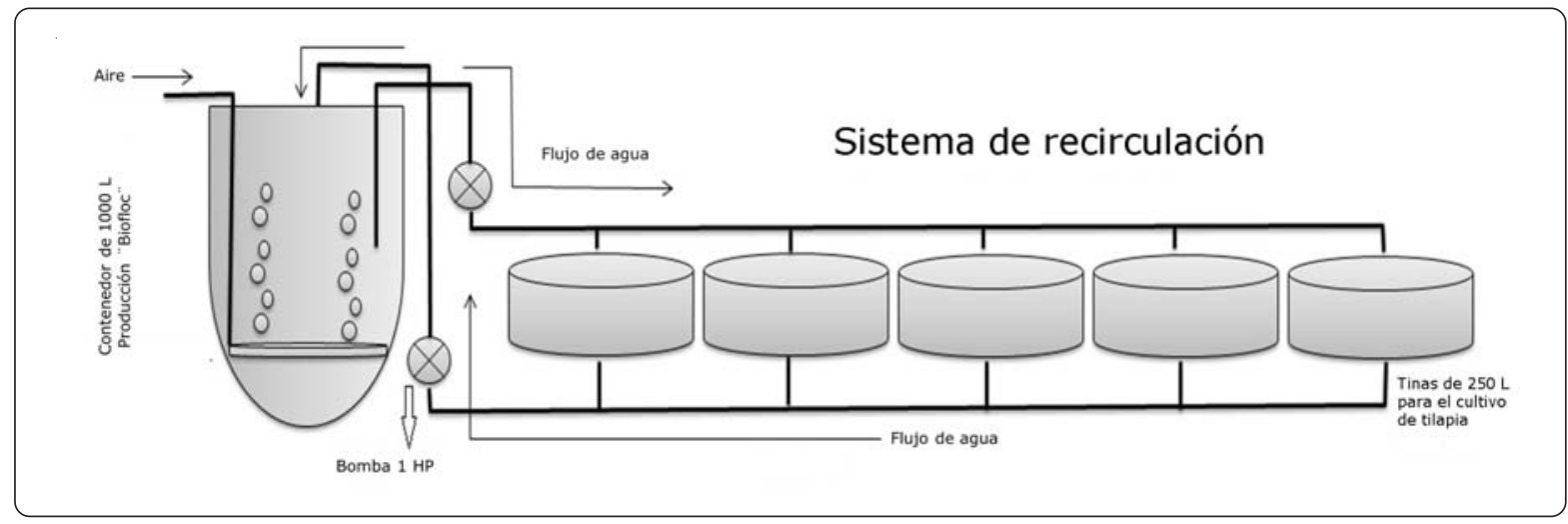

Figura 1. Sistema de recirculación utilizado en la producción de biofloc / Recirculation system used in the production of biofloc 


\section{CONFORMACIÓN DE BIOFLOC}

En la primera sección (cilindro de 1000 L), se colocaron 75 tilapias con una longitud de $5,0 \pm 0,95 \mathrm{~cm}$ y un peso promedio de 4,2 $\pm 1,08 \mathrm{~g}$, a las cuales se les proporcionó diariamente un alimento comercial formulado para la especie (Alimentos del Pedregal ${ }^{\circledR}$ ) con $45 \%$ de proteína y un tamaño de 0,6-0,8 mm (migaja 1), suministrando el $10 \%$ de su masa corporal y ajustando la cantidad cada 15 días.

Para garantizar un crecimiento bacteriano adecuado, se mantuvo una relación C/N= 15:1 (Avnimelech 2012), mediante el aporte controlado de carbono (melaza y pulido de arroz) y nitrógeno proveniente del alimento comercial. Esta condición fue mantenida durante 14 semanas.

\section{MoNitoReo de LAS COMUNIDADES MICROBIANAS}

\section{Aislamiento baCteriano}

Para realizar el conteo y caracterización de las comunidades bacterianas se utilizó el método propuesto por APHA(1992). A partir de la formación de los pequeños flóculos en el sistema, cada semana se tomaron muestras de $2 \mathrm{~g}$ que se inocularon en $90 \mathrm{~mL}$ de solución salina estéril y se efectuaron diluciones 1:10, de las cuales se sembró 0,1 mL en placas de agar MSR (Man-Rogosa Sharpe), BHI (Infusión Cerebro-Corazón), TCBS (Tiosulfato-CitratoSales Biliares) y TSA (Tripticasa-soja), por triplicado. Las placas se incubaron a $27^{\circ} \mathrm{C}$ durante $24 \mathrm{~h}$. Transcurrido este periodo, se efectuó el conteo de las unidades formadoras de colonias/mL y se caracterizó la morfología colonial; posteriormente a través de resiembras sucesivas las cepas fueron purificadas. La tinción de Gram se utilizó para observar la morfología celular con ayuda de un microscopio óptico (ZSX50 Olympus®). Este procedimiento se llevó a cabo durante 14 semanas.

A las cepas aisladas se les efectuaron pruebas microbiológicas convencionales para su identificación como: catalasa, oxidasa, óxido fermentación, movilidad e indol (Bergey \& Holt 1994). Finalmente, se confirmó la identificación de las cepas con el sistema API20E, API20NE, APICHL, API CHL 50 y el Programa Apiweb ${ }^{\text {TM }}$ Biomerieux.

\section{DIVERSIDAD DE LAS MICROALGAS Y MICROFAUNA ASOCIADA AL BIOFLOC}

Para la observación y cuantificación microscópica de los organismos asociados a los flóculos microbianos, se tomaron muestras de agua cada semana. En el caso de las microalgas se transfirió $1 \mathrm{~mL}$ de muestra a una cámara Sedgewick-Rafter (Azim \& Little 2008), y se observó con un microscopio ZSX50 Olympus ${ }^{\circledR}$, con objetivo de 100X conectado a un programa de imágenes; se procedió a contabilizar 4 campos escogidos al azar de la cámara de recuento.

Para la identificación de la microfauna (ciliados, rotíferos, nematodos) se tomaron 3 muestras de $10 \mathrm{~mL}$ de agua que se fijaron con formalina al $5 \%$, las cuales se observaron y contabilizaron de manera directa. Los microscopios utilizados estaban conectados al programa de imágenes y de conteo (Image ${ }^{\circledR}$ Pro Plus 7.0). La identificación taxonómica de los grupos observados se efectuó a nivel de género con ayuda de literatura especializada (Aladro-Lubel 2009).

\section{Resultados}

Los resultados obtenidos indican variaciones entre las diversas comunidades de organismos que se encuentran asociados al biofloc, dentro de los principales grupos observados se encuentran: microalgas, ciliados, rotíferos, nematodos, bacterias y la levadura Rhodotorula sp. (Fig. 2, Tabla 1).

\section{BACTERIAS Y LEVADURAS}

En la tabla 2 se muestran las especies microbianas identificadas. A partir de la tercera semana, se identificaron habitantes comunes del ambiente acuático como son los géneros Aeromonas y Vibrio que después de la séptima semana no fueron detectados en el sistema. En la cuarta semana, se establecieron comunidades microbianas heterótrofas con alta capacidad de degradar materia orgánica, como bacterias de los géneros Sphingomonas, Pseudomonas y Bacillus y la levadura Rhodotorula sp. La bacteria filamentosa Microthrix sp. relacionada con la formación de flóculos se identificó en la quinta semana. Entre los grupos bacterianos que dominaron el sistema después de la sexta semana se encontraron bacterias encargadas de la transformación del nitrógeno en el ambiente acuático como Nitrospira sp. y Nitrobacter sp. y Bacillus sp.

\section{Microalgas}

A partir de la tercera semana se inició la colonización de microalgas en el sistema, aún cuando no se observó la formación de flóculos (Fig. 3). El primer grupo que apareció fue el de las clorofitas que permanecieron hasta la semana 14 del experimento. Sin embargo, son las diatomeas las 
Tabla 1. Valor promedio ( $\pm \mathrm{DE}$ ) de los organismos encontrados en el biofloc durante 14 semanas de experimentación / Average value ( \pm SD) of the organisms found in biofloc during 14 weeks of experimentation

\begin{tabular}{cccccc}
\hline $\begin{array}{c}\text { Semana de } \\
\text { experimentación }\end{array}$ & Microalgas & Ciliados & Rotíferos & Nematodos & Bacterias \\
\hline 1 & 0 & 0 & 0 & 0 & 0 \\
2 & 0 & 0 & 0 & 0 & 0 \\
3 & $43 \pm 7$ & $13 \pm 2$ & $28 \pm 4$ & 0 & $620 \pm 65$ \\
4 & $115 \pm 10$ & $23 \pm 3$ & $35 \pm 5$ & $25 \pm 2$ & $4110 \pm 162$ \\
5 & $160 \pm 14$ & $33 \pm 3$ & $50 \pm 5$ & $46 \pm 5$ & $4650 \pm 150$ \\
6 & $283 \pm 24$ & $39 \pm 3$ & $54 \pm 1$ & $102 \pm 10$ & $5520 \pm 199$ \\
7 & $447 \pm 34$ & $39 \pm 3$ & $96 \pm 9$ & $125 \pm 13$ & $5600 \pm 273$ \\
8 & $513 \pm 37$ & $38 \pm 3$ & $91 \pm 10$ & $90 \pm 9$ & $5530 \pm 298$ \\
9 & $572 \pm 42$ & $32 \pm 3$ & $63 \pm 6$ & $90 \pm 9$ & $6910 \pm 411$ \\
10 & $527 \pm 46$ & $29 \pm 2$ & $46 \pm 6$ & $96 \pm 10$ & $6510 \pm 381$ \\
11 & $483 \pm 43$ & $29 \pm 2$ & $35 \pm 6$ & $95 \pm 10$ & $6810 \pm 372$ \\
12 & $517 \pm 46$ & $29 \pm 3$ & $41 \pm 7$ & $89 \pm 9$ & $6700 \pm 389$ \\
13 & $547 \pm 48$ & $28 \pm 3$ & $40 \pm 7$ & $90 \pm 9$ & $6970 \pm 400$ \\
14 & $493 \pm 43$ & $29 \pm 3$ & $39 \pm 7$ & $90 \pm 9$ & $6820 \pm 396$ \\
\hline
\end{tabular}
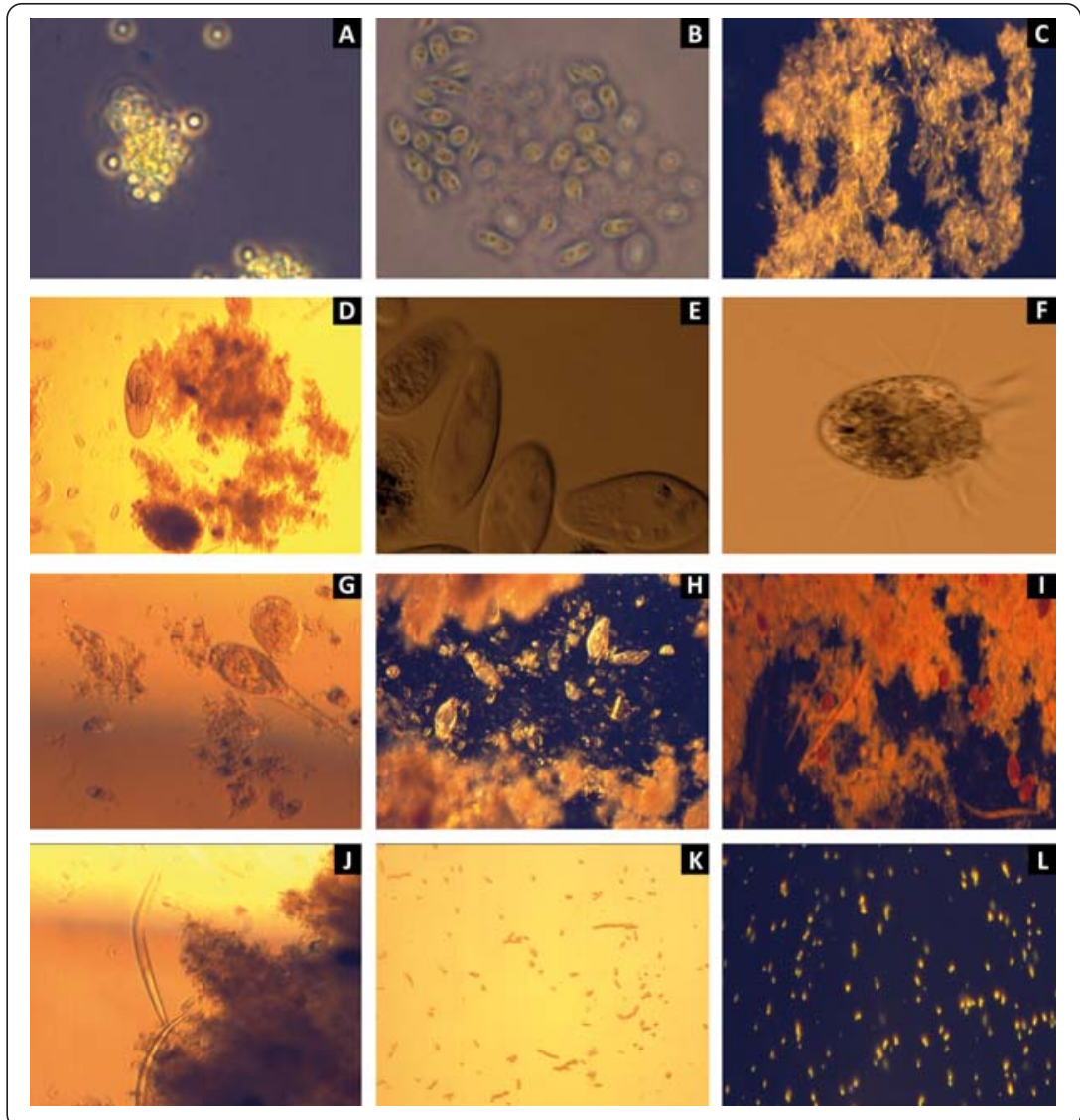

Figura 2. Composición de microorganismos observados en el biofloc. A) Algas clorofitas (40x); B) Cianobacterias (40x); C) Diatomeas (10x); D) Ciliado del género Paramecium (40x); E) Ciliado del género Colpidium (100x); F) Ciliado del género Halteria (100x); G) Rotíferos y ciliados (40x); H) Rotíferos del género Philodina (40x); I) Rotíferos y nematodos adheridos a los flóculos bacterianos (40x); J) Nematodos (40x); K) Vibrio sp. (40x); L) Levadura Rhodotorula sp. (40x) / Microorganisms composition observed at biofloc. A) Chlorophytes algae $(40 x)$; B) Cyanobacteria (40x); C) Diatoms (10x); D) Ciliate Paramecium genus (40x); E) Ciliate Colpidium genus (100x); F) Ciliate Halteria genus (100x ); G) Rotifers and ciliates (40x); H) Rotifers Philodina genus (40x); I) Rotifers and nematodes attached to the bacterial flocs (40x); J) Nematodes (40x); K) Vibrio sp. (40x); L) Yeast Rhodotorula sp. (40x) 
Tabla 2. Bacterias y levaduras identificadas en los flóculos durante 14 semanas de experimento / Bacteria and yeast identified in the flocs during 14 weeks of experiment

\begin{tabular}{|c|c|c|c|c|c|c|c|c|c|c|c|c|c|c|}
\hline \multirow{3}{*}{ Microorganismos } & \multicolumn{14}{|c|}{ Conteos $\left(\times 10^{4}\right.$ cel $\left.\mathrm{mL}^{-1}\right)$} \\
\hline & \multicolumn{14}{|c|}{ Semana de experimentación } \\
\hline & 1 & 2 & 3 & 4 & 5 & 6 & 7 & 8 & 9 & 10 & 11 & 12 & 13 & 14 \\
\hline Aeromonas hydrophila & $--\cdot$ & --- & 230 & 130 & 110 & 50 & 20 & $--\cdot$ & --- & --- & --- & --- & --- & --- \\
\hline Aeromonas salmonicida & --- & --- & 130 & 90 & 50 & 100 & 40 & --- & --- & --- & --- & --- & --- & --- \\
\hline Aeromonas sobria & --. & --. & 100 & 170 & 230 & 140 & 20 & --.- & --- & --- & --. & --. & 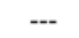 & --- \\
\hline Vibrio fluvialis & -- &.-- & 80 & 90 & 150 & 230 & 10 &.-- & -- & --- & $--\cdot$ & $--\cdot$ & --- & -- \\
\hline Vibrio vulmificus & -- & --- & 50 & 110 & 110 & 90 & 20 & --- & --- & --- & --- & --- & --- & --- \\
\hline Enterobacter sakazakii & -- & --- & 30 & 120 & 70 & 60 & 10 & --- & --- & --- & --- & --- & --- & --- \\
\hline Nitrospira sp. & --. & --- & --- & --- & --- & 58 & 675 & 110 & 105 & 121 & 1005 & 98 & 115 & 103 \\
\hline Bacillus subtilis & -- & --- & --- & 450 & 360 & 340 & 470 & 650 & 860 & 800 & 780 & 650 & 560 & 670 \\
\hline Bacillus sp. & --- & --- & --- & --- & 105 & 200 & 525 & 560 & 530 & 578 & 600 & 725 & 800 & 820 \\
\hline Sphingomonas paucimobilis & $\cdots$ & --- & --- & 420 & 390 & 560 & 550 & 560 & 160 & 84 & --- & --- & --- & -- \\
\hline Pseudomonas luteola & --- & --- & --- & 380 & 420 & 460 & 580 & 650 & 750 & 450 & 560 & 650 & 780 & 450 \\
\hline Pseudomonas mendocina & -- & --- & --- & 270 & 340 & 450 & 230 & 170 & 340 & 300 & 320 & 350 & 300 & 300 \\
\hline Microthrix sp. & -- & --- & --- & 340 & 450 & 560 & 650 & 340 & 230 & 250 & 320 & 300 & 350 & 350 \\
\hline Nitrobacter sp. & -- & --- & --- & 20 & 150 & 190 & 250 & 380 & 540 & 500 & 650 & 250 & 450 & 450 \\
\hline Micrococcus sp. & -- & --- & --- & --- & 410 & 520 & 480 & 350 & 490 & 450 & 400 & 380 & 400 & 400 \\
\hline Alcaligenes sp. & -- & --- & --- & --- & 150 & 240 & 340 & 250 & 65 & 23 & --- & --- & --- & --- \\
\hline Rhodotorula sp. & -- & --- & --- & --- & 34 & 65 & 780 & 750 & 1200 & 1080 & 1000 & 1050 & 1120 & 1090 \\
\hline
\end{tabular}

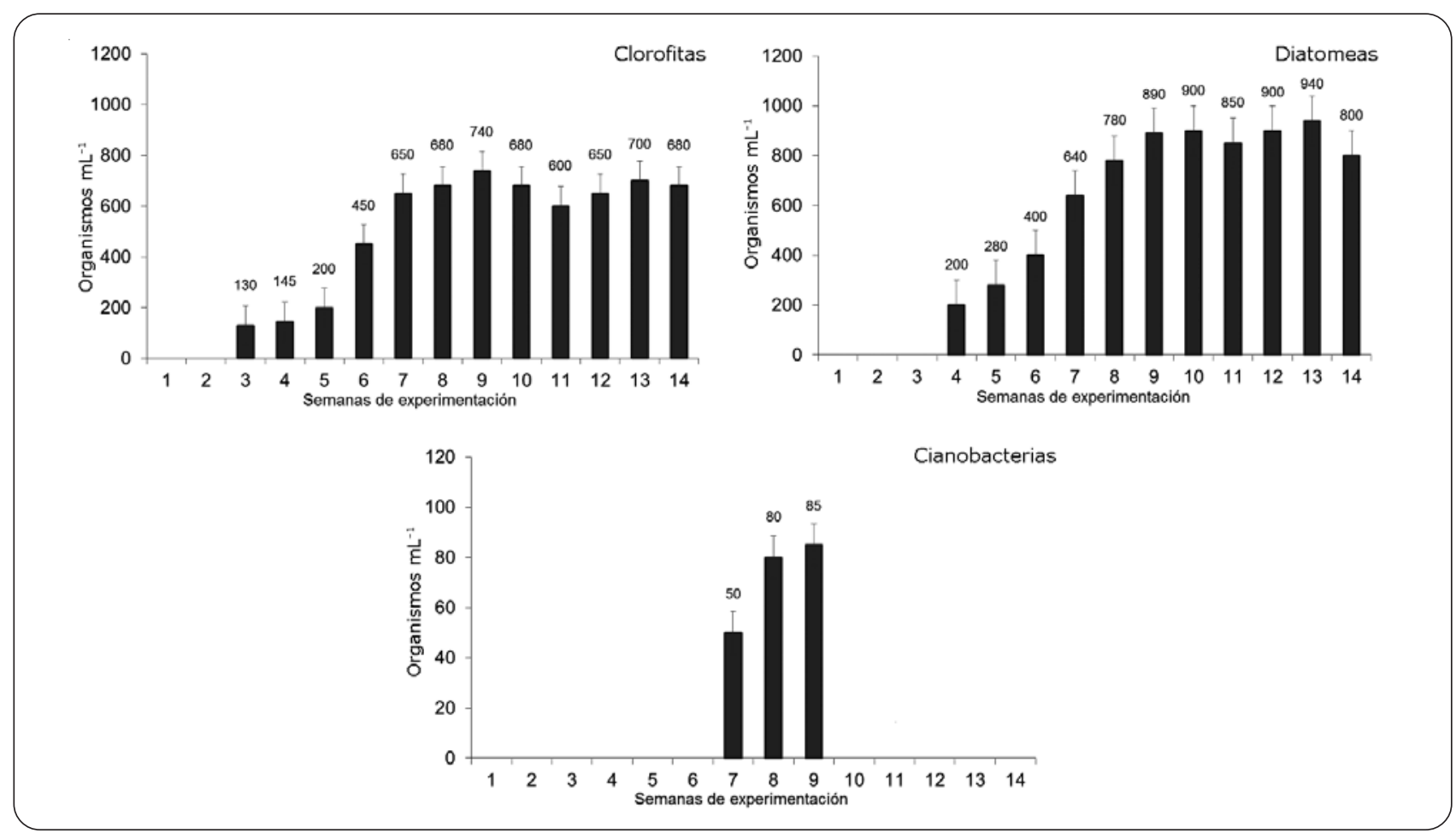

Figura 3. Abundancia de microalgas identificadas en el biofloc. Las barras corresponden a la desviación estándar / Abundance of microalgae identified in biofloc. Bars correspond to standard deviation 
que mantienen conteos mayores a partir de la octava semana y hasta el final del experimento. Es importante señalar que las diatomeas siempre se observaron muy adheridas a los flóculos. Las cianobacterias sólo estuvieron presentes entre la séptima y novena semana con conteos inferiores a 80 cel $\mathrm{mL}^{-1}$.

\section{Ciliados}

En relación a los ciliados se observó que los géneros que aparecieron a partir de la tercera semana fueron Paramecium, Colpidium y Stylonychia, permaneciendo Colpidium hasta el final del experimento (14 semanas).
Los géneros Vorticella, Epystilis y Halteria sólo se mantienen de 5 a 10 semanas (Fig. 4).

\section{RotífEROS}

Entre los rotiferos, el género Philodina fue el grupo dominante a lo largo del experimento, con conteos iniciales menores de 20 org. $\mathrm{mL}^{-1}$ pero incrementándose de tal manera que para la sexta semana alcanzaron una población de casi 200 org. $\mathrm{mL}^{-1}$ para después estabilizarse con conteos superiores a 115 org. $\mathrm{mL}^{-1}$ (Fig. 5).

El género Lecane apareció a partir de la tercera semana (80 org. $\mathrm{mL}^{-1}$ ), permaneciendo hasta la séptima (24 org. $\mathrm{mL}^{-1}$ ), cuando el género Keratella se incrementa (45 org.

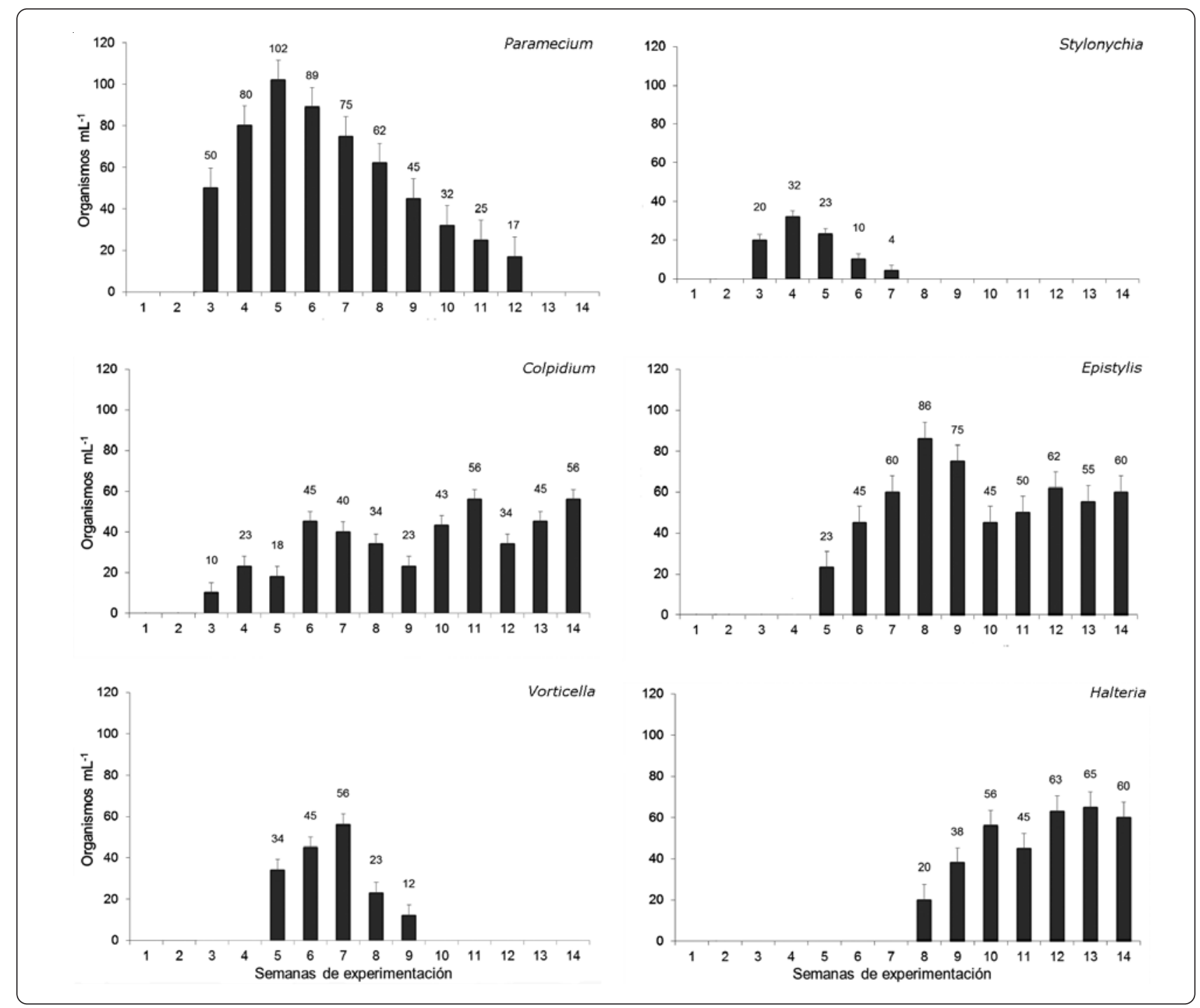

Figura 4. Grupos de ciliados observados en el biofloc. Las barras corresponden a la desviación estándar / Ciliates groups observed in biofloc. Bars correspond to standard deviation 


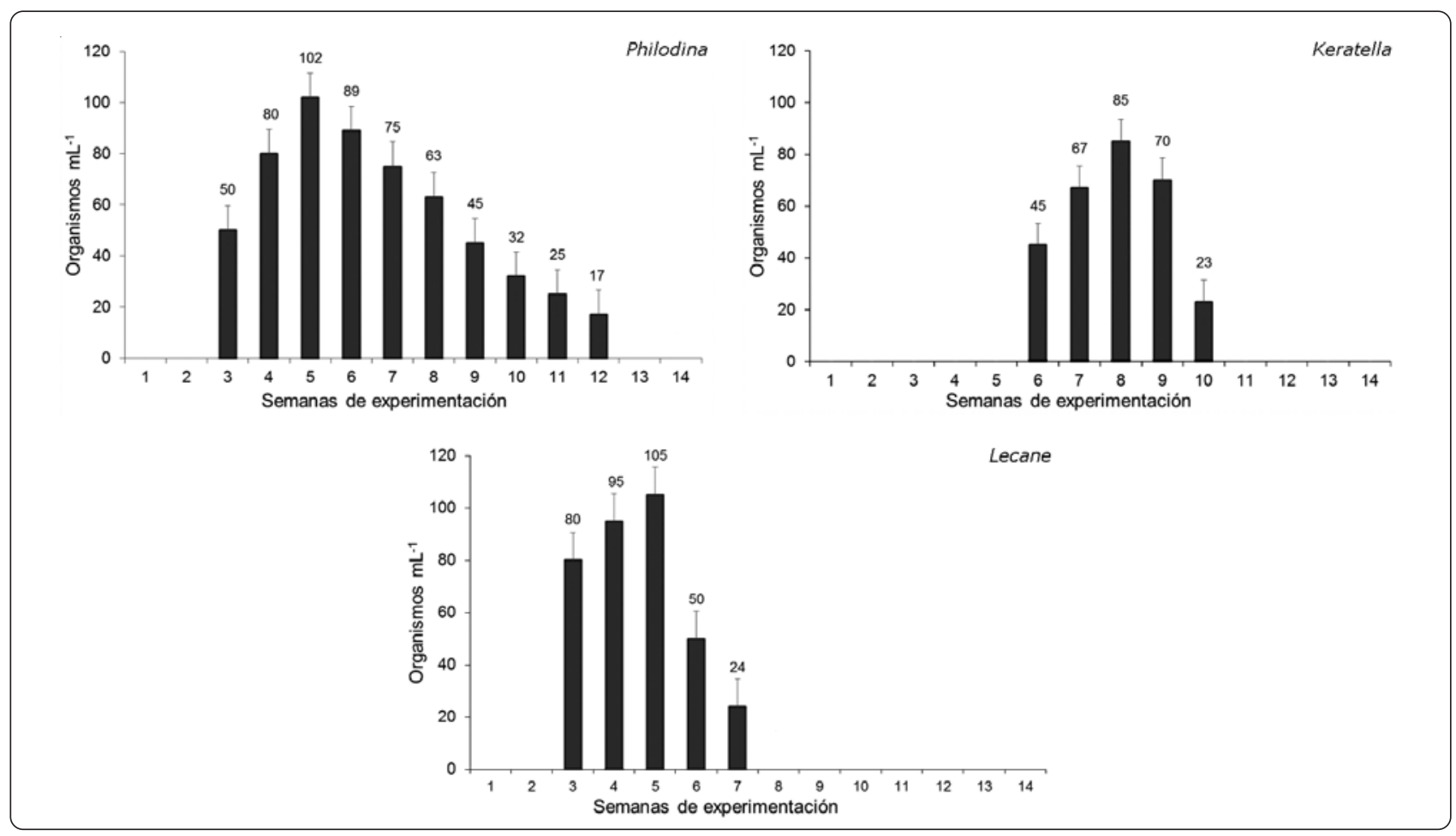

Figura 5. Abundancia de rotíferos identificados en el biofloc. Las barras corresponden a la desviación estándar / Abundance of rotifers identified in biofloc. Bars correspond to standard deviation

$\mathrm{mL}^{-1}$ ), pero este último sólo se registró durante 5 semanas, con una concentración final de 23 org. $\mathrm{mL}^{-1}$.

\section{Nematodos}

En la cuarta semana del experimento se observó la presencia de nematodos con una densidad de 25 org. $\mathrm{mL}^{-1}$, justo cuando los flóculos alcanzaron aproximadamente 1 mm de diámetro. El pico máximo fue observado en la séptima semana (125 org. $\mathrm{mL}^{-1}$ ), para estabilizarse en el sistema en las semanas posteriores (Fig. 6).

\section{Discusión}

Los resultados obtenidos en este estudio indican variaciones entre las diversas comunidades de organismos que se encuentran asociadas al biofloc. En relación a la composición bacteriana adherida a los flóculos, los primeros géneros identificados fueron Aeromonas y Vibrio que son habitantes comunes de ambientes acuáticos y proliferan sobre todo si hay sobrecarga de materia orgánica (Austin et al. 1995, Fuentes \& Pérez 1998). La problemática con estos géneros es que frecuentemente se muestran virulentos y causan enfermedades en diversos organismos acuáticos (Álvarez \& Austin 2000). Sin embargo, el incremento de microorganismos heterótrofos como Sphingomonas paucimobilis, Pseudomonas luteola, Pseudomonas mendocina, Bacillus sp. Micrococcus sp. y la levadura Rhodotorula sp., posiblemente impidieron la proliferación de los géneros Aeromonas y Vibrio de tal manera que no se encontraron después de la octava semana. Este comportamiento de disminución e inclusive desaparición, también lo señalan Wu et al. (2012), quienes mencionan que uno de los beneficios en el uso del biofloc es la capacidad de exclusión competitiva que tienen ciertas poblaciones bacterianas heterótrofas sobre bacterias patógenas.

La presencia de las bacterias degradadoras de nitrógeno como Nitrospira sp., Nitrobacter sp. y Bacillus sp., es benéfico, porque estos géneros están asociados a un mejor mantenimiento de la calidad del agua de cultivo Hargreaves (2006). Avnimelech et al. (1999, 2009) y Crab et al. (2012), señalan que estos grupos son dominantes en este tipo de sistemas, logrando un biocontrol efectivo sobre microorganismos patógenos. 


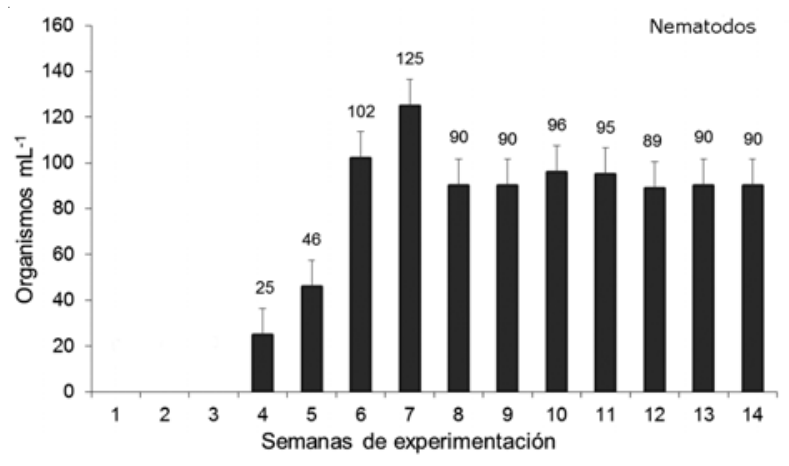

Figura 6. Dinámica del crecimiento de nematodos asociados al biofloc. Las barras corresponden a la desviación estándar / Growth dynamics of nematodes associated with biofloc. Bars correspond to standard deviation

En relación al género Bacillus, se tiene información que posee propiedades probióticas, ya que secretan una gran variedad de exoenzimas y polímeros que generan un ambiente hostil a bacterias, sobretodo patógenas (Hong et al. 2005, Ziaei-Nejad et al. 2006, Monroy et al. 2010). Entre los polímeros secretados, destaca el poli-ßhidroxibutirato (PHB), que es un exo-polisacárido con efecto probiótico similar a los ácidos orgánicos (Sinha et al. 2008) y puede representar hasta un $80 \%$ de la materia seca de células bacterianas y un 16\% de la materia seca del biofloc (De Schryver et al. 2012). Este exo-polisacárido es sintetizado en mayor cantidad cuando la bacteria presenta condición de estrés fisiológico o nutricional (Sinha et al. 2008). Al lograr la exclusión de microrganismos dañinos, se logra un mayor bienestar a los organismos cultivados. Al parecer este comportamiento sucedió en el presente experimento, en donde el género Bacillus apareció en la cuarta semana y su efecto se manifestó en la octava semana cuando las bacterias patógenas fueron excluidas.

En el caso de las microalgas, se observó que aunque las clorofitas iniciaron la colonización del biofloc, fue el grupo de las diatomeas el que dominó después de la octava semana, mientras que las cianobacterias sólo fueron observadas de la sexta a la novena semana. Contrariamente, otros autores señalan que las clorofitas son las dominantes sobre las diatomeas en este tipo de sistemas (Ray et al. 2010). Estas diferencias pueden deberse a la cantidad y calidad de nutrientes contenidos en el medio y al consumo selectivo que tienen ciertas especies de ciliados y rotíferos que controlan a las poblaciones microalgales (Kuang et al. 2004). Maicá et al. (2012), observaron que en un sistema biofloc utilizado en cultivo de juveniles de Litopenaeus vannamei, se presentaron mayores cantidades de clorofitas a salinidad de $2 \mathrm{~g} \mathrm{~L}^{-1}$ y de diatomeas a $25 \mathrm{~g} \mathrm{~L}^{-1}$. Así, Riquelme \& Avendaño-Herrera (2003), mencionan la importancia de la relación microalga-bacteria como determinante de los grupos que se desarrollan en los ambientes acuáticos, de tal manera que el aumento de bacterias heterótrofas transformadoras de carbono, posibilitan un incremento de diatomeas en el sistema y restringe a otros grupos como las cianobacterias, tal como sucedió en esta investigación.

Los ciliados son considerados un rico alimento natural de peces y camarones (Castro et al. 2004). En este estudio los ciliados estuvieron representados por 6 géneros: Paramecium, Stylonychia, Vorticella, Colpidium, Epistylis y Halteria, en comparación con el trabajo de Loureiro et al. (2012), donde identificaron solamente 3 géneros: Unorema, Litonotus y Euplotes en el cultivo de camarón con sistema biofloc. Los autores señalan que los elementos que producen los flóculos tales como la fuente de carbono, el alimento balanceado, así como los peces acondicionados para el sistema, pueden tener una influencia directa sobre los grupos de organismos que se desarrollan. Ballester et al. (2010) observaron concentraciones mínimas y máximas de ciliados de 39 y 169 org. $\mathrm{mL}^{-1}$, respectivamente, en cultivos de postlarvas de Farfantepenaeus paulensis en sistemas de biofloc durante 45 días. Maicá et al. (2012) observaron concentraciones promedio de ciliados de 164, 64 y 29 org. $\mathrm{mL}^{-1}$ en aguas de salinidad 2, 4 y $25 \mathrm{~g} \mathrm{~L}^{-1}$, respectivamente. En el presente estudio (14 semanas) realizado en agua dulce, se observaron valores mínimos y máximos de 10 y 102 org. $\mathrm{mL}^{-1}$, respectivamente, observando que la salinidad y el tiempo de cultivo pueden afectar la concentración de ciliados en el agua.

Con respecto a los rotíferos, se observaron los primeros organismos a partir de la tercera semana, identificando los géneros Lecane, Keratella y Philodina, siendo éste ultimo el que predominó después de la séptima semana. Loureiro et al. (2012), indican que los rotíferos frecuentemente están asociados al biofloc. Esto se debe a que los rotíferos pueden fragmentar los flóculos y consumir las bacterias adheridas, además que el mucílago producido por sus excreciones también ayuda a la formación de nuevos flóculos (Pérez 2010). Ballester et al. (2010), observaron concentraciones mínimas y máximas de rotíferos de 4,6 y $151 \mathrm{org}$. $\mathrm{mL}^{-1}$, respectivamente, en agua marina $\left(35 \mathrm{~g} \mathrm{~L}^{-1}\right)$. En el presente estudio, las concentraciones observadas fueron de 5 y $196 \mathrm{org}$. $\mathrm{mL}^{-1}$, bastante similar a un ambiente marino. 
Otro grupo importante dentro del biofloc son los nematodos, sobre todo por presentar altos contenidos de proteína cruda y ácidos grasos esenciales en su composición (De Lara 2005). Éstos se observaron a partir de la cuarta semana con valores promedio de $25 \mathrm{org}$. $\mathrm{mL}^{-1}$, obteniendo un pico máximo a la séptima semana (125 org. $\mathrm{mL}^{-1}$ ) para estabilizarse en el sistema en las semanas posteriores. Ray et al. (2010), mencionan que los nematodos son uno de los grupos más importantes en los sistemas biofloc y que su abundancia está determinada por la presencia de diversos ciliados (e.g., Paramecium y Colpidium) que le sirven de alimento. Además, la tasa de consumo de los peces que se encuentran incluidos en el sistema puede igualmente intervenir en su concentración. Focken et al. (2006) y Loureiro et al. (2010) reportan la presencia de nematodos en el contenido estomacal de peces cultivados en el sistema biofloc y sugieren que son una rica fuente de alimento vivo in situ y disponible las $24 \mathrm{~h}$.

Los resultados de esta investigación aportan avances en el conocimiento sobre la composición de diferentes bacterias y levaduras que se encuentran asociados al biofloc y que influyen en el mejoramiento de la calidad del agua y el bienestar de los organismos en cultivo, al identificarse grupos bacterianos señalados con anterioridad como probióticos. Además, la descripción de los cambios en las comunidades de microalgas, ciliados, rotíferos y nematodos a lo largo del periodo de experimentación, permite reconocer la contribución del biofloc como fuente de alimento natural in situ, que es tan importante en la dieta de peces como de crustáceos de importancia comercial sobretodo en estadios larvarios.

\section{LITERATURA CITADA}

Aladro-Lubel M. 2009. Manual de protozoarios, 123 pp. Facultad de Ciencias, Universidad Autónoma de México, México.

Álvarez J \& B Austin. 2000. Especies de Vibrio y Aeromonas aisladas del intestino de camarones marinos sanos, silvestres y cultivados en Venezuela. Veterinaria Tropical 25(1): 527.

APHA. 1992. Standard methods for examination of water and waste-water, 1011 pp. American Public Health Association, Washington.

Austin B, LF Stuckey, PAW Robertson, I Effendi \& DRW Griffith. 1995. A probiotic strain of Vibrio alginolyticus effective in reducing diseases caused by Aeromonas salmonicida, Vibrio anguillarum and Vibrio ordalii. Journal of Fish Diseases 18(1): 93-96.
Avnimelech Y. 1999. Carbon nitrogen ratio as a control element in aquaculture systems. Aquaculture 176(3-4): 227-235.

Avnimelech Y. 2006. Bio-filters: The need for a new comprehensive approach. Aquacultural Engineering 34(3): 172-178.

Avnimelech Y. 2009. Biofloc technology: a practical guide book, $181 \mathrm{pp}$. The World Aquaculture Society, Baton Rouge.

Avnimelech Y. 2012. Biofloc technology -a practical guide book, 272 pp. The World Aquaculture Society, Baton Rouge.

Azim ME \& DC Little. 2008. The biofloc technology (BFT) in indoor tanks: water quality, biofloc composition, and growth and welfare of Nile tilapia (Oreochromis niloticus). Aquaculture 283(1-4): 29-35.

Ballester ELC, PC Abreu, RO Cavalli, M Emerenciano, L Abreu \& W Wasielesky. 2010. Effect of practical diets with different protein levels on the performance of Farfantepenaeus paulensis juveniles nursed in a zero exchange suspended microbial flocs intensive system. Aquaculture Nutrition 16: 163-172.

Bergey D \& JG Holt. 1994. Bergey’s manual of determinative bacteriology, 787 pp. Williams \& Wilkins, Baltimore.

Castro MG, BT Castro \& MJ Castro. 2004. Protozoarios en: alimento vivo para organismos acuáticos, 129 pp. AGT Editor, México.

Crab R, M Kochva, W Verstraete \& Y Avnimelech. 2009. Bio-flocs technology application in over-wintering of tilapia. Aquaculture Engineering 40: 105-112.

Crab R, T Defoirdt, B Peter \& W Verstraete. 2012. Biofloc technology in aquaculture: Beneficial effects and future challenges. Aquaculture 356-357: 351-356.

De Lara AR. 2005. Panagrellus redivirus (Nematoda) cultivado en medio de avena enriquecido con Spirulina sp. para probar el crecimiento de la población y calidad nutritiva. Tesis de maestría, Facultad de Ciencias, Universidad Nacional Autónoma de México, México, 72 pp.

De Schryver P, R Crab, T Defoirdt, N Boon \& W Verstraete. 2008. The basics of bio-flocs technology: the added value for aquaculture. Aquaculture 277: 125-137.

De Schryver P, N Boon, W Verstraete \& P Bossier. 2012. The biology and biotechnology behind bioflocs. In: Avnimelech Y (ed). Biofloc technology - a practical guide book, pp. 199-215. The World Aquaculture Society, Baton Rouge.

Ekasari J, R Crab \& W Verstraete. 2010. Primary nutritional content of bio-flocs cultured with different organic carbon sources and salinity. Hayati Journal of Bioscience 17: 125130.

Emerenciano M, ELC Ballester, RO Cavalli \& W Wasielesky. 2011. Effect of biofloc technology (BFT) on the early postlarval stage of pink shrimp Farfantepenaeus paulensis: growth performance, floc composition and salinity stress tolerance. Aquaculture International 19: 891901. 
Emerenciano M, ELC Ballester, RO Cavalli \& W Wasielesky. 2012. Biofloc technology application as a food source in a limited water exchange nursery system for pink shrimp Farfantepenaeus brasiliensis (Latreille, 1817). Aquaculture Research 43: 447-457.

Emerenciano M, G Gaxiola \& G Cuzon. 2012. Biofloc technology applied to shrimp broodstock. In: Avnimelech Y (ed). Biofloc technology -a practical guide book, pp. 217-230. The World Aquaculture Society, Baton Rouge.

Emerenciano M, G Cuzon, M Arevalo \& G Gaxiola. 2013. Biofloc technology in intensive broodstock farming of the pink shrimp Farfantepenaeus duorarum: spawning performance, biochemical composition and fatty acid profile of eggs. Aquaculture Research January 2013, <doi: 10.1111/ are.12117>

Focken U, C Schlechtriem, WM Von, OA García, CA Puello \& K Becke. 2006. Panagrellus redivivus mass produced on solid media as live food for Litopenaeus vannamei larvae. Aquaculture Research 37: 1429-1436.

Fuentes RJM \& HJA Pérez. 1998. Isolation of Aeromonas hydrophila in the rainbow trout (Oncorhynchus mykiss) Veterinaria Mexico 29: 117-119.

Hargreaves JA. 2006. Photosynthetic suspended-growth systems in aquaculture. Aquaculture Engineering 34: 344-363.

Hong HA, LH Duc \& SM Cutting. 2005. The use of bacterial spore formers as probiotics. FEMS Microbiology Reviews 29: 813-835.

Kuang Q, Y Bi, Y Xia \& Z Hu. 2004. Phytoplankton community and algal growth potential in Taipinghu reservoir, Anhui Province, China. Lakes \& Reservoirs: Research \& Management 9: 119-124.

Loureiro KC, WJ Wilson \& PC Abreu. 2012. Utilização de protozoários, rotíferos e nematódeos como alimento vivo para camarões cultivados no sistema BFT. Atlântica, Rio Grande 34(1): 5-12.

Maicá PF, MR Borba \& W Wasielesky. 2012. Effect of low salinity on microbial floc composition and performance of Litopenaeus vannamei (Boone) juveniles reared in a zerowater-exchange super-intensive system. Aquaculture Research 43: 361-370.

Monroy DMC, BT Castro, PFJ Fernández \& RL Mayorga. 2010. Inhibition of Aeromonas hydrophila by probiotic strains isolated from the digestive tract of Pterophyllum scalare. Revista Mexicana de Ingeniería Química 9(1): 37-42.
Newall P, N Bate \& LA Metzeling. 2006. Comparison of diatom and macroinvertebrate classification of sites in the Kiewa River system. Hydrobiologia 572(1): 131-149.

Pérez AJD. 2010. Aplicación y evaluación de un reactor de contactores biológicos rotativos (RBC o biodiscos), a escala de laboratorio como tratamiento de los lixiviados generados en el relleno sanitario de la Pradera. Tesis de Maestría Ingeniería Urbana, Facultad de Ingenierías, Universidad de Medellín, Medellín, 259 pp.

Ray AJ, BL Lewis, CL Browdy \& JW Leffler. 2010. Suspended solids removal to improve shrimp (Litopenaeus vannamei) production and an evaluation of a plant-based feed in minimal-exchange, superintensive culture systems. Aquaculture 299(1-4): 89-98.

Riquelme C \& H Avendaño. 2003. Interacción bacteriamicroalga en el ambiente marino y su potencial uso en acuicultura. Revista Chilena de Historia Natural 76: 725736.

Sinha AK, K Baruah \& P Bossier. 2008. Horizon scanning: the potential use of biofloc as an anti-infective strategy in aquaculture -an overview. Aquaculture Health International 13: 8-10.

Timmons MB, JM Ebeling, FW Wheaton, ST Sommerfelt \& BJ Vinci. 2002. Microbial biofloc and protein levels in green tiger shrimp. Recirculating aquaculture systems, 748 pp. Caruga Aqua Ventures, New York.

Wasielesky W, H Atwood, A Stokes \& CL Browdy. 2006. Effect of natural production in a zero exchange suspended microbial floc based super-intensive culture system for white shrimp Litopenaeus vannamei. Aquaculture 258: 396403.

Wu L, C Peng, Y Peng, L Li, S Wang \& Y Ma. 2012. Effect of wastewater $\mathrm{COD} / \mathrm{N}$ ratio on aerobic nitrifying sludge granulation and microbial population shift. Journal Environment Science 24(2): 234-241.

Ziaei-Nejad S, RM Habibi, TG Azari, DL Lovett, AR Mirvaghefi \& M Shakouri. 2006. The effect of Bacillus spp. bacteria used as probiotics on digestive enzymes activity, survival and growth in the Indian white shrimp Fenneropenaeus indicus. Aquaculture 252: 516-524.

Recibido el 9 de abril de 2013 y aceptado el 3 de octubre de 2013

Editor: Claudia Bustos 